\title{
Descartes-Snell law of refraction with absorption
}

\author{
S.A. Kovalenko \\ Institute of Semiconductor Physics, NAS Ukraine, 45 Prospect Nauki, Kyiv, 03028, Ukraine
}

\begin{abstract}
The state of the art in the theory of optical constants of matter is considered for different spectral ranges of light absorption. It is stressed that up to now no there exists no commonly accepted formula for calculation of refractive index in the X-ray region. Starting from three different approaches, an analysis is made of relations between the angles of refraction and incidence in the case of a transparent medium-absorbing medium interface. Through analysis of the corresponding plots each of relations is estimated from the standpoint of possibility for its practical application. A novel version of the Descartes-Snell law is advanced. For the first time an expression (15) is obtained that is completely substantiated, both mathematically and physically. It may be recommended for use, first of all, when calculating multiplayer coatings in the X-ray optics units. It is stated that further investigations in this area are required, especially when performing experiments for different regions of optical and X-ray spectra.
\end{abstract}

Keywords: absorbing medium, law of light refraction, x-ray optics, optical constants.

Paper received 12.01.01; revised manuscript received 27.02.01; accepted for publication 13.07.01.

\section{Review of literature and formulation of the problem}

1. The law of refraction for light rays has been established by Snell and Descartes as long ago as the first half of XVII century. However, determination of an angle of refraction for a ray passing through a boundary between transparent and strongly absorbing media still makes a problem. In this case the refractive index $\tilde{n}_{2}$ of the latter medium is complex, so the angle of refraction is complex too. The Descartes-Snell law of refraction is:

$$
\frac{\sin \alpha}{\sin \beta}=\frac{\tilde{n}_{2}}{n_{1}},
$$

where $\alpha(\beta)$ is the angle of incidence (refraction).

Presence of a complex function in expression (1) does not permit to determine a direction of the light ray propagation in the medium 2. In some cases (say, when solving various problems of $X$-ray optics whose elements are based on the refraction phenomenon) it is of importance to know this direction. The real values of refractive indices in the Xray spectral region are close to unity. Therefore at sizable angles of incidence a refracted ray will propagate near the surface where optical properties of the material may substantially differ from those in the bulk.
2. The situation is complicated by the fact that in the physical community up to now there is no universally accepted concept of the refractive index for solids in the $\mathrm{X}$-ray spectral region. Some of physicists believe that it is over unity while others have directly opposite opinion. To illustrate, in the monograph [1] by Lorentz it is stated that

$$
n=1-\delta=1-\frac{N e \lambda^{2}}{2 \pi m c^{2}} \text {. }
$$

Here $N$ is the number of atoms per $1 \mathrm{~cm}^{3} ; \lambda$ is the wavelength of light; $c$ is the speed of light in vacuum; $e(m)$ is the electron charge (mass); $\delta=(1.68 \pm 0.08) \cdot 10^{-6}$. Since all the parameters in the second term on the righthand side of expression (2) are positive, the refractive index $n<1$. It is stated in [2] that expression (2) agrees rather well with experiment for wavelengths below any intrinsic resonance absorption.

In [3] Ewald wrote: «The ordinary dispersion formule can thus be applied to the high-frequency case. They tell us that the optical density $n^{2}-1$, which is of the order of unity for visible light, is of the order of $10^{-5} \cdot 10^{-6}$ for soft and hard X-rays, respectively. This smallness is the reason why Röntgen and many others after him found no reflection or refraction of X-rays». So, by Ewald, $n^{2}-1 \approx$ $\approx 10^{-5} \div 10^{-6}$, i.e., $n^{2}=1+10^{-5} \cdot 10^{-6}$ and $n>1$. 


\section{S.A. Kovalenko et al.: Descartes-Snell law of refraction with absorption}

In the monograph [4] by Baryshevsky, an expression for X-ray refractive index is of the following form:

$$
n=1+\frac{2 \pi \rho}{k^{2}} .
$$

Here $\rho$ is the scattering material density; $f(0)$ is the amplitude of elastic coherent scattering through zero angle; $k=2 \pi / \lambda$. Since these quantities are positive, $n>1$.

In the monograph [5] by Compton and Allison the complex refractive index is represented as

$$
\tilde{n}=1-\frac{N e}{2 \pi m v^{2}}-i \beta
$$

i.e., its real part is just the right-hand side of the Lorentz formula (2), since $v^{2}=c^{2} / \lambda^{2}$; so $n<1$.

The fourth volume of the Physical Encyclopedia published in 1965 [6] gives the Lorentz formula for the refractive index, i.e., $n<1$. However, in the Physical Encyclopedia published in 1994 [7] the following expression is given:

$$
n=1+4 p N a \text {. }
$$

Here $N$ is the number of particles (atoms, molecules) per $\mathrm{cm}^{3}$ and $\alpha$ is their polarizability. Both quantities are positive. So $n$ is over unity for all electromagnetic waves. Without setting off the X-ray region, the author stresses that the secondary coherent waves, «interfering with the wave incident on the medium, form a resulting light wave that is propagating in the medium with a phase speed $c_{1}<c$, and so $n=c / c_{1}>1 »$.

The authors of [8] made an analysis of a possibility to obtain Cerenkov radiation in the $\gamma$-range of the electromagnetic scale. To estimate this possibility, they started from the expression for refractive index obtained in [9]:

$$
n=1+\frac{\lambda^{2}}{2 \pi} N f=1+\frac{\lambda^{2}}{2 \pi}\left(f^{R}+f^{N}\right)
$$

Here $f^{R}=-Z r_{e}$ is the Rayleigh scattering amplitude; $Z$ is the atomic number; $r_{e}$ is the classical electron radius. The X-ray frequencies lie over all the atomic resonance frequencies, and the amplitude of Rayleigh scattering on atoms is negative. Therefore the real part of refractive index is less than unity and, as the authors of [8] state, the Cerenkov radiation cannot occur in the X-ray spectral region. The situation, however, is quite different in the $g$ range if one takes into account the coherent scattering by nuclei (i.e., the function $f^{N}$ ). A negative contribution to unity (stemming from the Rayleigh amplitude $f^{R}$ ) in expression (6) could be counterbalanced (with an excess) by a positive contribution from the amplitude of $\gamma$-quanta resonance scattering. In this case $n$ becomes bigger than unity.

An interesting situation seems to take place: the Cerenkov effect, that can be realized in both $\gamma$-range and the whole optical region, cannot occur in the $\mathrm{x}$-ray region that presents a very wide frequency range between the $\gamma$ and optical radiation. Nevertheless, a pessimism concer- ning X-ray efficiency for Cerenkov radiation emission seems to have no solid ground. In recent years some reports have appeared [10] on positive results in this area. (This, of course, does not remove the problems related to determination of ray propagation after passing the transparent medium-absorbing medium interface.) This follows from an analysis of many works dealing with one of the most important problems of optical instrumentation designing X-ray optics units. To illustrate, see the materials of the conference organized by the Russian Academy of Sciences that have been published in [11]; all of them are representative of the state-of-the art in this area.

3. Researches dealing with refractometry of the nearsurface layers in solids are of great importance in the context of the discussed problem. In [12] a detailed description is given of a new method for investigation of surfaces. This method has been put to a test when studying single-crystalline gallium arsenide and semiconductor heterostructures of $\mathrm{Ge}_{x} \mathrm{Si}_{1-x} / \mathrm{Si}$-type.

The starting points of the method were the law of refraction in the form

$\sin \phi_{1} / \sin \phi_{2}=n_{2} / n_{1}=1 /(1-\delta-i \beta)$

and formula for the $s$-component of Fresnel reflection coefficient

$R_{s}=\left(\frac{E_{s}}{E_{0 s}}\right)^{2}=\frac{(Q-a)^{2}+b^{2}}{(Q+a)^{2}+b^{2}}$,

where

$$
\begin{aligned}
& a^{2}=\frac{1}{2}\left\{\left[\left(\theta_{1}^{2}+2 \delta\right)^{2}+4 \beta^{2}\right]^{\frac{1}{2}}+\theta_{1}^{2}+2 \delta\right\}, \\
& b^{2}=\frac{1}{2}\left\{\left[\left(\theta_{1}^{2}+2 \delta\right)^{2}+4 \beta^{2}\right]^{\frac{1}{2}}-\theta_{1}^{2}-2 \delta\right\}
\end{aligned}
$$

The following designations are used in expressions (7)-(9): $n_{2}=1$ is the refractive index of vacuum (air); $n_{1}=$ $1-\delta-i \beta$ is the complex refractive index of the material studied; $E_{s}\left(E_{0 s}\right)$ is $s$-component of the electric vector of light wave in the material (air). The authors suppose that for grazing incidence (Fig. 1) at angles $\theta_{1} \leq 1^{\circ}$ the reflection coefficient for $p$-component will be the same.

The angles in Fig. 1 are related to those of incidence and refraction in expressions (1), (7) in the following way:

$\theta_{1}=\frac{\pi}{2}-\phi_{1}, \theta_{2}=\frac{\pi}{2}-\phi_{2}$,

and the deviation angle (between the refracted and incident rays) is

$\theta_{3}=\theta_{2}-\theta_{1}$.

The surprising thing is that expressions (8) and (9) used by the authors of [12] differ from, as they write, the expressions that are widely used in the case of total exter- 


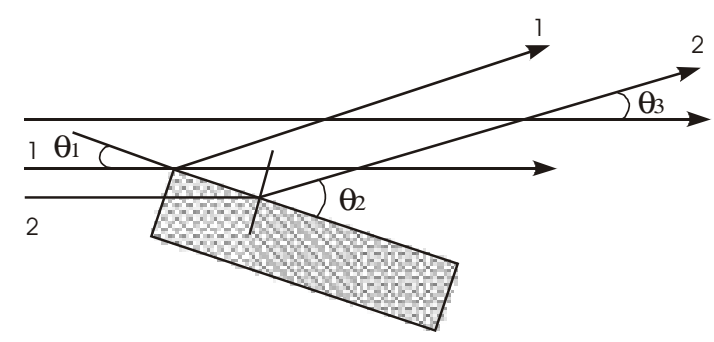

Fig. 1. Geometry of ray propagation for incidence on the sample side face $(1,2)$ and leaving into the air $\left(1^{\prime}, 2^{\prime}\right)$.

nal reflection in the signs of auxiliary variables $a$ and $b$, and this fact essentially affects the character of the angular dependence of the reflection coefficient $R\left(\theta_{1}\right)$. In such a situation the authors of [12] should substantiate both the validity of their choice of signs and incorrectness of the alternative one. It remains incomprehensible why they did not make this.

In [12] the deviation angle $\theta_{3}$ is formally written down in complex form as

$$
\theta_{3}=\theta_{2}-\theta_{1}=\sqrt{\theta_{1}^{2}+2 \delta+2 i \beta}-\theta_{1} .
$$

For estimation of the effect of its imaginary part (without knowing law of refraction) the authors referred to the formule used in metal optics [13] to determine a contribution from the imaginary part of $n_{1}$ to angular shift of the refracted ray. According to their estimation, it was about $0.1 \%$ and therefore has not been taken into account.

4. The question of what is the formation depth for a reflected light ray in any electromagnetic spectral range also belongs to the discussed problem. This is of particular importance at strong absorption, because in this case the near-surface layers of a sample may play a great part. Such a problem has been recently considered for silicon with oxide thickness varying over its surface [14]. By a depth $D$ of ray penetration into a medium the authors of [14] meant such a distance along the normal to the surface over which the electromagnetic wave intensity dropped by a factor of $e$ [15]:

$$
\frac{1}{D}=2 \frac{\omega}{c} \operatorname{Im} \sqrt{E-\cos ^{2} \theta_{0}} \text {. }
$$

Here $\theta_{0}$ is the angle of grazing incidence; $e$ is the material permittivity.

According to expression (11), a rise of the angle of grazing incidence (i.e., a drop of the angle of incidence) is accompanied by an increase in the penetration depth for electromagnetic wave. This dependence is qualitatively supported by experiment. However, the numerical discrepancy between the theoretical and experimental values is significant: the experimental values exceed the calculated ones by a factor of $1.3 \div 3.0$. (When we say about a qualitative agreement, we mean that $D$ grows with $\theta_{0}$.) The experimental and theoretical curves differ significantly: while experiment gives a linear dependence over the whole range of angles $\theta_{0}$ used, the theoretical curve does not show change of $D$ up to $\theta_{0}=5^{\circ}$. The authors attribute this discrepancy to the «non-Fresnel» character of the system studied, its anisotropy due to the buried $\mathrm{SiO}_{2}-\mathrm{Si}$ interface or inadequate account of light absorption by material in expression (11). They hope to study a reason for the above discrepancies in more detail during further investigations.

5. The above review indicates at a need for further development of the theory of optical properties of materials in the range of intense light absorption, in particular, determination of an expression for the Descartes-Snell law in the case of a transparent medium-absorbing medium interface. This problem is solved and discussed in the next section.

\section{Law of refraction with absorption}

1. The first efforts to get a formula for the law of refraction in an absorbing medium have been made in [16]. For the electromagnetic wave polarized perpendicularly to the plane of incidence ( $s$-component) it was obtained that the law of refraction is of the same form as in the case of transparent media. This result looks surprising, because it states that light absorption by the second medium has no effect on the angle of refraction.

A different result has been obtained in [16] for the component polarized in the plane of incidence ( $p$-component):

$\frac{\sin \alpha}{\sin \beta}=\frac{n_{2}\left(1-\frac{k_{2}^{2}}{n_{2}^{2}}\right)}{n_{1}}$,

where $k_{2}$ is the absorption coefficient for the second medium. (The first medium was taken to be transparent, as in the previous case.)

From expression (12) it follows that if the refractive index equals to the absorption coefficient, then the numerator of the fraction in the right-hand side of expression (12) is zero, so that angle of refraction remains undetermined at any angle of incidence. That is why the author of [16] believes that $k$ has to lie below $n$.

2. Quite different result can be obtained using the replacement $n \rightarrow n+i k$. In this case

$$
\frac{\sin \alpha}{\sin \beta}=\frac{\sin \alpha}{\sin \left(\beta^{\prime}+i \beta^{\prime \prime}\right)}=\frac{n_{2}+i k_{2}}{n_{1}} \text {. }
$$

Using Euler formule, one gets

$$
\begin{aligned}
& \sin ^{2} \beta^{\prime}=\frac{1}{2}\left[\left(1+\frac{n_{1}^{2} \sin ^{2} \alpha}{n_{2}^{2}+k_{2}^{2}}\right)-\right. \\
& -\sqrt{\left.\left(1+\frac{n_{1}^{2} \sin ^{2} \alpha}{n_{2}^{2}+k_{2}^{2}}\right)^{2}-\frac{4 n_{2}^{2} n_{1}^{2} \sin ^{2} \alpha}{\left(n_{2}^{2}+k_{2}^{2}\right)^{2}}\right]} .
\end{aligned}
$$




\section{S.A. Kovalenko et al.: Descartes-Snell law of refraction with absorption}

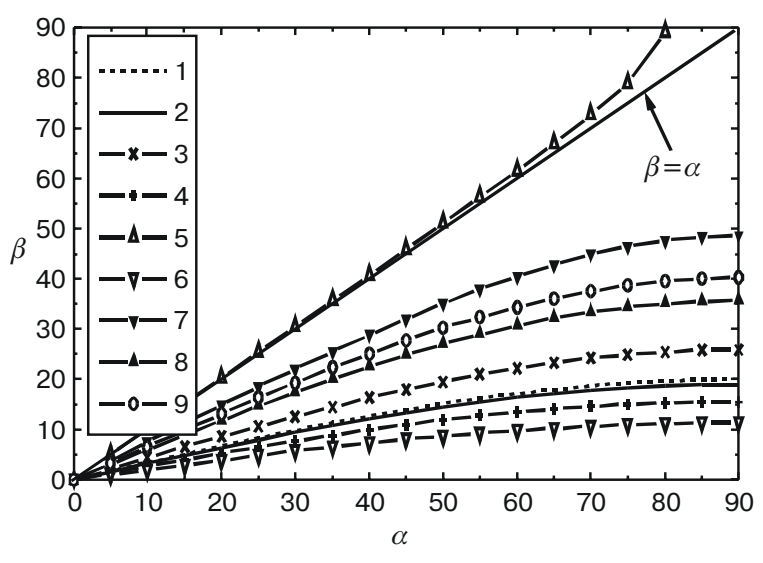

Fig. 2. Angle of refraction $\beta$ vs angle of incidence $\alpha$ curves calculated from expressions (12) (curves 1, 3, 5, 7), (14) (curves $2,4,6,9)$ and (15) (curve 8) for solids with different optical parameters: $n_{2}=3, k_{2}=0.4587(1,2) ; n_{2}=3, k_{2}=1.4587(3,4) ; n_{2}$ $=3, k_{2}=2.4587(5,6) ; n_{2}=1.5, k_{2}=0.5(7,8,9)$.

This expression determines direction of light propagation in an absorbing medium for two ( $s$ - and $p$-) components that are polarized in mutually perpendicular planes. In a uniform isotropic medium both components propagate in the same direction, and not in different ones.

3. Yet another approach has been used in [17]. After writing down the equations for the plane of equal phases and plane of equal amplitudes, we get the following expression for the sine of refraction angle:

$$
\sin \beta=\frac{\sqrt{2} \sin \alpha}{\sqrt{n_{2}^{2}-k_{2}^{2}+\sin ^{2} \alpha+\sqrt{\left(n_{2}^{2}-k_{2}^{2}-\sin ^{2} \alpha\right)^{2}+4 n_{2}^{2} k_{2}^{2}}}}
$$

In the case when a ray passes through the interface between two transparent media the two last-named formulae give the traditional interrelation (that has long been known) following from the law of refraction. We mention in passing that the interrelation (12) also reduces to the traditional law of refraction in the case when there is no light absorption in the second medium.

4. One would think that the above result could serve as validity criterion for expressions (14) and (15). However, the plots of interrelation between the angles $\alpha$ and $\beta$ at fixed $n$ and $k$ values indicate at discrepancies between the data obtained from expressions (14), (15).

Shown in Fig. 2 are (i) three pairs of curves (the set of the optical constants $n$ and $k$ being the same for each pair) plotted using expressions (12) and (14) and (ii) three curves (for the same set of $n$ and $k$ values) plotted using expressions (12, (14) and (15). It is immediately apparent that the angle of refraction goes up monotonically with the angle of incidence in all the cases but one, namely, curve 5 plotted from expression (12). This curve is characterized by practical coincidence of the angles $\beta$ and $a$ in the $0^{\circ}<\alpha<40^{\circ}$ range. A possible reason for this could be that the contribution to the angle of refraction from absorption coefficient (though $k$ being less than $n$ ) is sufficient enough to compensate for that from the index of refraction. Expression (12) is not valid for $\alpha \geq 80^{\circ}$. In general the domain of definition for the function (12) depends on $k$ value. This fact must not take place in actual conditions. The above domain is presented in Fig. 3. The boundary values are as follows:

$k_{1}=\left[\left(n_{2}-n_{1}\right) / n_{1}\right]^{\frac{1}{2}}$ and $k_{2}=\left[\left(n_{2}+n_{1}\right) / n_{1}\right] \frac{1}{2}$.

Related to curve 5 discussed above is curve 6 . It is plotted using the same $n$ and $k$ values (as in the case of curve 5) but from expression (14). A distinction between the two curves is such compelling that one intuitively wishes to give preference to curve 6 that (contrary to curve 5 ) is not apart from the rest of curves.

The curves in Fig. 2 belonging to the first pair practically coincide. Those from the second pair (they correspond to the same refractive index value, but absorption coefficient is about three times greater than for the first pair) demonstrate a considerable discrepancy (up to $10^{\circ}$ at grazing incidence). It should be noted that this discrepancy has the same character as in the first pair. If the absorption coefficient is increased by a factor of five, then the difference between the boundary angles of incidence is about $80^{\circ}$ (curves 5 and 6 ).

By and large, from correlation between all the curves presented in Fig. 2 one can conclude that, at the same set of parameters $n$ and $k$, the biggest values of the angle of refraction are those given by expression (12), while expression (14) gives the smallest ones.

The angle of refraction vs angle of incidence curves calculated from expressions (14) and (15) are given in Fig. 4. Two pairs of curves are plotted using the optical parameters of zirconium at light wavelengths $243.0 \AA$ (curves 1,2) and $135.5 \AA$ (curves 3, 4). Other two pairs of

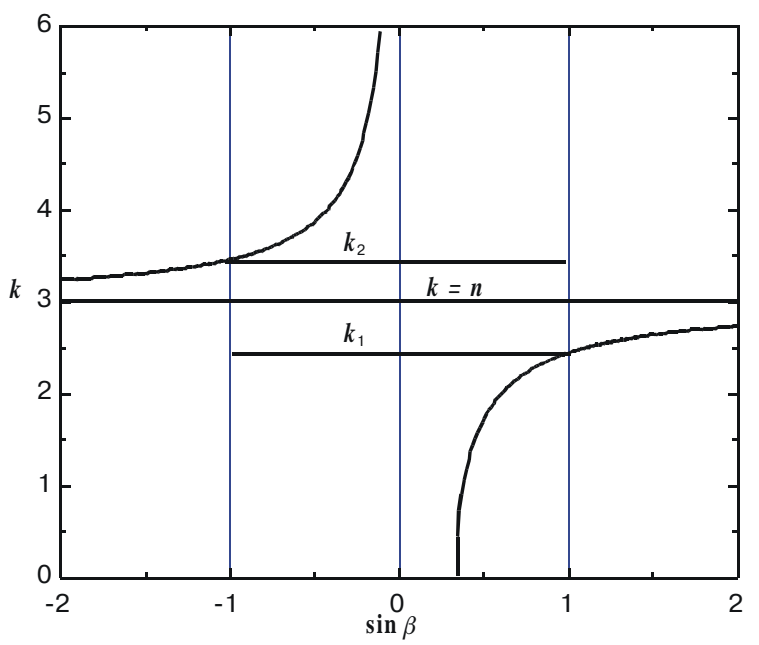

Fig. 3. Domain of definition for the function (12) at fixed value $n=3$. 


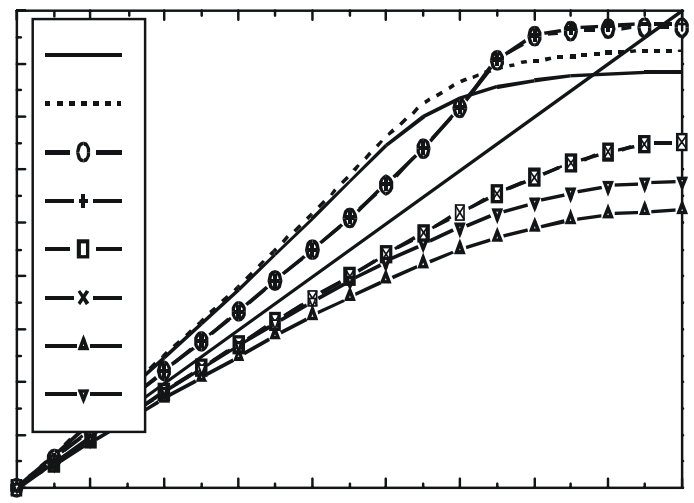

Fig. 4. Angle of refraction $\beta$ vs angle of incidence $\alpha$ curves for zirconium $(1,2-\lambda=243.0 \AA, 3,4-\lambda=135.5 \AA)$ and an arbitrary solid (5-8) calculated from expressions (14) (curves 1, 3, 5, 7) and (15) (curves 2, 4, 6, 8): $n_{2}=0.808, k_{2}=0.0987(1,2) ; n_{2}=0.95$, $k_{2}=0.01(3,4) ; n_{2}=1.10, k_{2}=0.02(5,6) ; n_{2}=1.10, k_{2}=0.20(7,8)$.

curves are plotted for a virtual solid with $n=1.10$ and $k=0.02$ or 0.20 . In two cases (curves 3,4 and 5,6 ) the expressions (14) and (15) give the same results, while in two other cases (curves 1, 2 and 7,8) the curves obtained from (15) lie below those calculated from expression (14).

\section{Conclusions}

Summing up, it should be noted that contributions to the angle of refraction value from the refractive index and absorption coefficient are not fully apparent. So one cannot formulate a corresponding rule and say a priori what of these two factors is predominant. However, it is possible to state that expressions (14) and (15) can be used in any spectral region. We give preference to expression (15) obtained by us. The reason for this is that the replacement $n \rightarrow n+i k$ is strictly substantiated in a mathematical sense, while determination of refraction angle between the planes of equal phases and equal amplitudes requires application of some assumptions. Besides, when the absorption coefficient is increased, the refractive index calculated from expression (15) changes stronger than that obtained using expression (14). This fact agrees well with changes in the optical properties of materials.

As for expression (12), it shouldn't be recommended for use because it is wrong.

\section{Acknowledgements}

The author is grateful to M.P. Lisitsa and G.G. Tarasov for fruitful discussions and help in work.

\section{References}

1. G.A. Lorentz, Theory of electron and application to the light occurrence and thermal radiation. 2-d edition, Moscow, (1953). (In Russian)

2. M.A. Blokhin, X-rays in Physic encyclopedia, 30. p. 468, IL, Moscow. (1960). (In Russian).

3. P.P. Ewald, Crystal optics for visible light and $\mathrm{x}$ rays // Rev. Mod. Phys. 37(1), pp. 46-56 (1965).

4. V.G. Baryshevsky, Nuclear optic of polarization environment, Minsk (1976) (In Russian).

5. A.H. Compton, S.K. Allison, X-Rays in Theory and Experiment, p.828, Princeton (1935).

6. Refraction of x-rays, in Physical encyclopedia dictionary 4, p. 191, Sovetskaya Entsiklopediya, Moscow (1965). (In Russian).

7. V.I. Malyshev, Refractive index, in Fizicheskaya Entsiklopediya 4, p.107. Bol'shaya Rossiyskaya Entsiklopediya, Moscow. (1994). (In Russian)

8. V.V. Fedotov, A.I. Smirnov, On a possibility of Cerenkov radiation of $\gamma$-quanta by electrons // Pis'ma ZhETF 23(1), pp.34-36 (1976). (In Russian)

9. R. Newton, The theory of scattering of wave and particles, Mir, Moscow, p. 607 (1969). (In Russian).

10. N.K. Zhevago, Induced Cerenkov radiation of soft X-rays. / / Nucl. instrum. and methods in phys. research. 331(1/3), p. 584 (1993).

11. Poverkhnost'. Rentgenovskie, Sinkhrotronnye i Neitronnye Issledovaniya, N 1 (Russian Working Conference on X-ray, 23-26 February 1998, Novgorod) (1999).

12. A.G. Tur'yansky, I.V. Pirshin, X-ray refractometry of surface layers // Pribory i Tekhnika Eksperimenta. N 6, pp. 104-111 (1999). (In Russian).

13. M. Born, E. Wolf, Principles of Optics, Pergamon Press (1980).

14. E.O. Filatova, A.S. Shulakov, V.A. Luk'yanov, Depth of formation of soft x-ray reflected beam at mirror reflection // Fiz. Tverd. Tela 40(7), pp. 1360-1363 (1998) (in Russian).

15. A.A. Eichenwald // Zhurn. Rossiiskogo Fiziko-Khimicheskogo Obschestva 41, p. 131 (1909). (In Russian).

16. T.A. Kudykina, Boundary conditions in case of electromagnetic wave absorption // Phys. Stat. Sol. (b). 160(1) pp. 365373 (1990); Opt. pop. of Semicond (Ge, Se, GaAs, InSb). 165, pp. 591-598 (1991).

17. A.V. Sokolov, Optical Properties of Metals, GIFML, Moscow (1961). (In Russian).

18. D.L. Windt, W.C. Cash, J.M. Scott, P. Arendt, B. Newnam, R.F. Fisher, A.B. Swartzlander, P.Z. Takacs, J.M. Pinneo, Optical constants for thin films of $\mathrm{Ti}, \mathrm{Zr}, \mathrm{Nb}, \mathrm{Mo}, \mathrm{Ru}, \mathrm{Rh}, \mathrm{Pd}$, $\mathrm{Ag}, \mathrm{Hf}, \mathrm{Ta}, \mathrm{W}, \mathrm{Re}, \mathrm{Ir}, \mathrm{Os}, \mathrm{Pt}$, and Au from $24 \mathrm{~A}$ to $1216 \mathrm{~A} / /$ Appl. Opt. 27, pp. 246-278 (1988). 